\title{
A Survey On Opportunistic Routing Protocols In Cellular Network For Mobile Data Offloading
}

\author{
Sanjit Kumar Dash ${ }^{1}$, Chandrakanta Mahanty ${ }^{2}$, Sasmita Mishra ${ }^{3}$ and Jibitesh Mishra ${ }^{1}$ \\ ${ }^{1}$ College of Engineering and Technology, Bhubaneswar, Odisha, India \\ ${ }^{2}$ C. V. Raman College of Engineering, Bhubaneswar, Odisha, India \\ ${ }^{3}$ Indira Gandhi Institute of Technology, Saranga, Odisha, India
}

\begin{abstract}
Currently cellular networks are overloaded, due to rapid growth of various smartphone applications. With the arrival of $3 G$ and $4 G$ network technologies mobile data traffic has been growing at an unprecedented pace and increasingly surpassing the network capacity. Mobile data offloading is an effective network congestion demotion approach to solve the overload issue through opportunistic communication. It is a beneficiary approach, because there is no monetary cost for it. Subscribe Send Architecture and Opportunistic forwarding protocol plays a vital role during offloading by allowing users to subscribe the contents but does not download the subscribed content on the Content Service Provider thereby network traffic reduces up to a greater extent. A quantitative study on performance evaluation of opportunistic routing protocols like Epidemic, Spray and Wait, ProPhet and MaxProp are discussed in this paper. We have compared these protocols based on few performance metrics like overhead ratio, delivery probability and average latency. Our simulation results verify that MaxProp performs well and can offload cellular traffic effectively.
\end{abstract}

\section{KEYWORDS}

Content service provider, mobile data offloading, opportunistic communication, opportunistic routing protocols, smartphones

\section{INTRODUCTION}

Mobile data offloading also called as Wi-Fi offloading is the use of complementary network communication technologies to deliver mobile data traffic originally planned for transmission over Wi-Fi networks [1]. Complementary network communication technologies include integrated mobile broadcast, Wi-Fi and fem to cell. The amount of data which should be carried out on the cellular bands can be reduced by offloading scheme and also freeing bandwidth for other users. The primary source of mobile data traffic includes different internet applications, from browsers to video and audio streaming applications processing in smartphone devices, feature phones, tablets and laptops with $3 \mathrm{G}$ access capabilities. The popularity of social media, video and demand of internet gaming across a wide variety of new devices like smartphones and tablets has created a rush of network data traffic. Quick growth of smartphones, laptops and tablets, data traffic on the networks is increasing and mobile connections are predicted to reach 7.4 billion marks by 2015 striking the global population [2]. According to Cisco's survey, monthly global mobile data traffic will exceed 24.3 Exabyte by 2019 [3].

Different offload options are available to offload data from the mobile network. The major work of mobile operator is to determine which option is best based on various factors such as

DOI: $10.5121 /$ ijwmn.2018.10103 
infrastructure, customer consumption patterns, distribution and maintenance cost, complexities and user density in a specific location. Six different mobile data offload options are [4]: $i$. Wi-Fi Hotspot, ii. LTE Small Cells,iii. Wi-Fi / Integrated Femto, iv. Direct Tunnel, v. Internet Offload Gateway (IOGW), vi. M2M Gateway

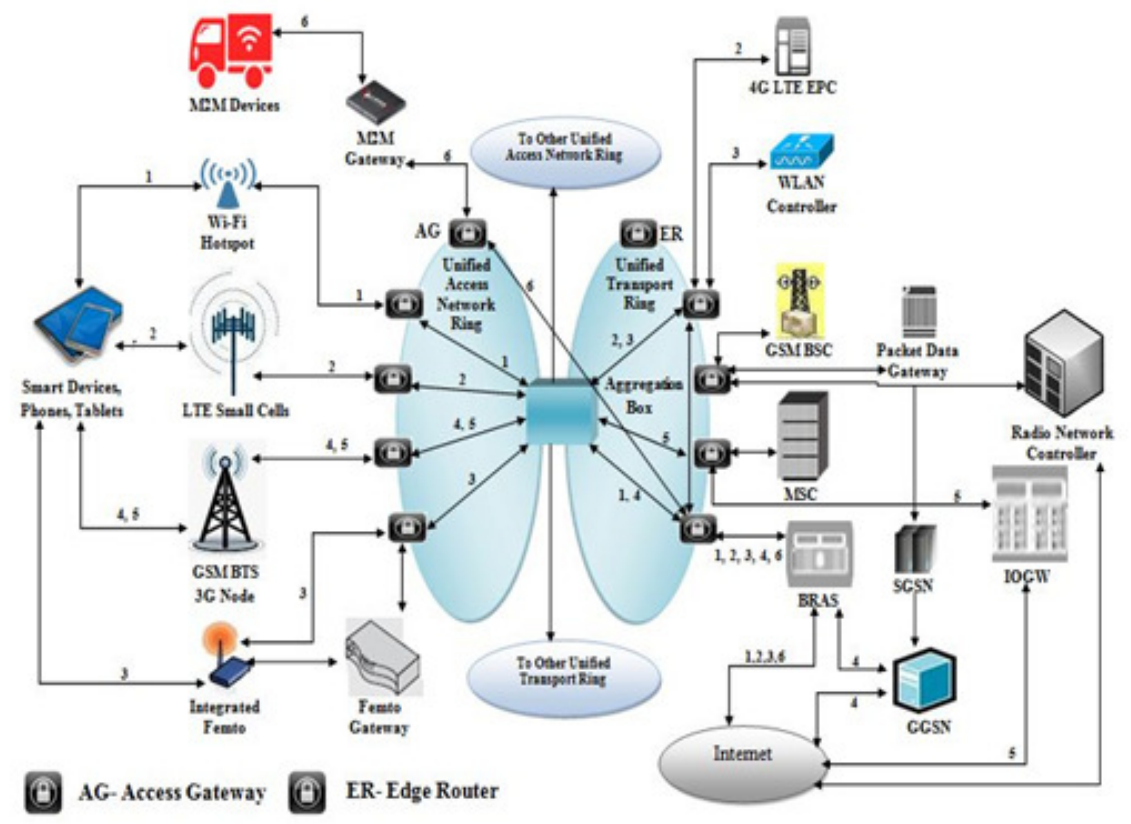

Figure 1: Mobile Data offloading Architecture Overview

\section{Subscribe And SEnd ArChitecture}

The data traffic from mobile network can be offloaded using Subscribe-and-Send architecture [7].The transmission model of subscribe send architecture deals with the point of spreading content to different set of smart mobile terminals (SMT) equipped with 3G. Each SMT subscribe contents on CSP and deliver information to encountering nodes with the help of software.

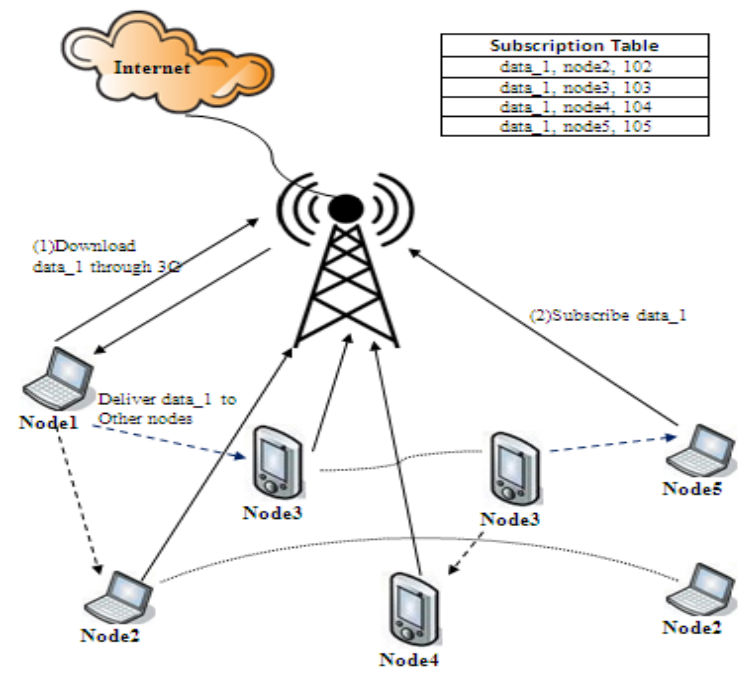

Figure 2: Subscribe and Send Architecture 
Subscribe-and-Send architecture works in two stages:

i. Subscribe stage- a user accesses the CSP and initiates subscription including name of the subscribed content, the user's ID and the time limit of the subscription.

ii. Send stage- source nodes are interested to download the contents through the mobile network.

At first a node accesses the contents to CSP and checks whether someone has subscribed to the content or not. If the node has content that is subscribed by other available nodes, it starts the forwarding operation and sends the content to the subscriber through opportunistic Wi-Fi routing. When two nodes encounter, they exchange their respective subscription tables. In figure 2, the solid path represents the link created by $3 \mathrm{G}$ interface and the dotted path represents peer-to-peer Wi-Fi communication.

\section{LITERATURE SURVEY}

There are several solutions for mobile traffic offloading as discussed below:

Wifler [8] can be used to augment $3 \mathrm{G}$ capabilities in cellular environments. Fast switching mechanism and leveraging delay tolerance concepts are used to overcome the performance and the poor availability of Wi-Fi. To predict Wi-Fi connectivity Wifler uses a plain replica of the environment. Wifler uses different kinds of predictions to offload more amounts of data on Wi-Fi, but only if delaying minimizes the $3 \mathrm{G}$ usage. The whole things can be successfully finished within the application's tolerance threshold. When Wi-Fi is not able to successfully dispatch the data packet within a little time span then Wifler change its control to $3 \mathrm{G}$ rapidly.

Line2 [9] is an app which is used to utilize Wi-Fi, 3G and $4 \mathrm{G}$ data connections in mobile network. At first Line2 attempts to connect via a Wi-Fi network. If Wi-Fi network is nonexistent, it will then seeks to link over $3 \mathrm{G}$ or $4 \mathrm{G}$ mobile data network giving the flexibility to the mobile without utilizing cell minutes. At last, it will connect via mobile connection, which will utilize cellular plan minutes.

iPass [10] system gives access to the iPass cellular network which is the world's vast trading WiFi network. Here users get the benefits of simple, effortless and reliable connectivity virtually anywhere they wander. Universal Wi-Fi network and trusted mobility services are given by iPass.

MOTO Project [11] is used to carry out the boundaries of 4G/LTE technologies in congested conditions. It also determines how opportunistic communication and networking can be used as a trustworthy way to offload some segment of the traffic from the 4G/LTE network. MOTO project is structured around five scientific and technological objectives: 1.To design an integrated operator-managed offloading system, 2. To design combined offloading algorithms, 3. To characterize the capacity benefits of the system, 4 . To perform fine-grained large scale evaluation, 5. To carry out integrated prototyping and trials. MOTO project has designed a proper model and implemented the latest upgrades in opportunistic networking to attain traffic offloading in a productive manner. So that overloading issues of cellular frameworks can be reduced. 
International Journal of Wireless \& Mobile Networks (IJWMN) Vol. 10, No. 1, February 2018

\section{Opportunistic Communications}

It is a method that at each time chooses the best among multiple communication alternatives in a network. The aim of opportunistic communication is to promote information distribution in the arising mobile social networks and thus by minimizing the load of mobile data traffic [5]. Opportunistic Networks is a network of wireless connected nodes. Nodes may be either mobile or fixed. Opportunistic Communication requires mobile nodes that are able to perform some communication with each other even if a route linking to the nodes never exists. Here nodes are not conscious about the network topology [6]. In this communication, expectations regarding the presence of a full path between two communicating nodes are never formed. So that, source and destination node are unable to present in the same network at the same time. Opportunistic communication approaches allow such nodes to interchange their data between them. Opportunistic Communication gives a profitable promise to commercial owners by offering zero expenses or immensely low cost framework requirements.

\section{Opportunistic Routing Protocol}

Opportunistic routing protocols take profit from broadcast transmission which permits multiple nodes to obtain the exact data packet with just one complete transmission. Few opportunistic routing protocols are discussed below:

1) Epidemic Routing (ER): ER is a flooding-based forwarding algorithm for DTNs proposed by Vahdat et al. [12]. In this, the node receiving a message, forwards a copy of it to all nodes it encounters. Thus, the message is spread throughout the network by mobile nodes and eventually all nodes will have the same data. The algorithm gives no guarantee in delivering the packets to the destination. Each message and its unique identifier are saved in the node's buffer. The list of them is called the summary vector. Whenever two adjacent nodes get opportunity to communicate with each other, they exchange and compare their summary vectors to identify which messages they do not have and subsequently request them. To avoid multiple connections between the same nodes, the history of recent contacts is maintained in the nodes caches.

2) Probabilistic Routing Protocol (PRoPHET): PRoPHET is developed by Lindgren et al. [13]. The basic assumption in the PRoPHET is that mobility of nodes is not purely random, but it has a number of deterministic properties e.g. repeating behavior. In the PRoPHET scheme, it is assumed that the mobile nodes tend to pass through some locations more than others, implying that passing through previously visited locations is highly probable. As a result, the nodes that met each other in the past are more likely to meet in the future. The first step in this method is the estimation of probabilistic metric called delivery predictability, $P(a, b) \in[0,1]$. This metric estimate the probability of the node $\mathrm{A}$ to be able to deliver a message to the destination node B. Whenever a node comes in to contact with other nodes in the network, they exchange summary vectors. The summary vectors also contain the delivery predictability values for destinations known by each node. Each node further requests messages it does not have and updates its internal delivery predictability vector to identify which node has greater delivery predictability to a given destination. 
3) Spray-and-Wait (S-n-W): Spyropoulos et al. proposed S-n-W protocol [14] with an objective to control the level of spreading of messages throughout the network. Spray and wait protocol assumes no knowledge of network topology and nodes mobility patterns and simply forwards multiple copies of messages received using flooding technique. The protocol spreads L copies of the message. The spray and wait method consists of two phases, spray phase and wait phase. In the spray phase the source node after forwarding L copies of message to the first $L$ encountered nodes, goes to the wait phase, waiting for delivery confirmation. In the wait phase all nodes that received a copy of the message wait to meet the destination node directly to deliver data to it. Once data is delivered confirmation is sent back using the same principle.

4) MaxProp: MaxProp proposed by Burgess $J$ et al. [15] is based on prioritizing both the schedule of packets transmitted to other peers and the schedule of packets to be dropped. These priorities are based on the path likelihoods to peers according to historical data, acknowledgments, a head-start for new packets, and lists of previous intermediaries. It replicates those messages that are not held by the encountered node. When a contact is discovered, all messages not held by the encountering node will be replicated and transferred to the encountering node. It has high computational complexity. It unifies the problem of scheduling of packets for transmission to other peers and determines which packets should be deleted when buffers are low on space.

\section{Simulation AND Results}

In this section, we compared MaxProp, Spray-and-Wait, Epidemic and PRoPHET protocol using Opportunistic Network Environment (ONE) simulator to estimate forwarding behavior, performance of traffic offloading, delivery probability, average latency, overhead ratio of these protocols. We use a simple map-based movement model as specified in ONE simulator [16]. The simulator considered six groups. Simulation work carried out for 120, 180, 240 nodes by dividing them into six equal size groups. The transmission range of all nodes is 100 meters and transmission speed is $10 \mathrm{MB}$ per second. The simulation should run for 5000 seconds. Figure 3 shows the comparison of delivery probability, average latency and overhead ratio of Epidemic, PRoPHET, Spray- and-Wait and MaxProp under different number of nodes.

As an overall summary to the above routing protocols, we have considered some of the crucial factors like Category, Delivery Probability, Average Latency, Overhead Ratio, Simulation Speed, RAM for JVM for differentiating the opportunistic routing protocols. The detail comparisons are given in Table-1.

Table 1: Comparison of Epidemic, Prophet, Spray-And-Wait and Maxprop Protocols

\begin{tabular}{|c|c|c|c|c|}
\hline $\begin{array}{l}\text { Comparison } \\
\text { Factor }\end{array}$ & Epidemic & ProPHET & $\begin{array}{c}\text { Spray- } \\
\text { n-Wait }\end{array}$ & $\begin{array}{l}\text { Max } \\
\text { Prop }\end{array}$ \\
\hline Categorization & Content Oblivious & $\begin{array}{c}\text { Partially } \\
\text { Content } \\
\text { Aware }\end{array}$ & $\begin{array}{c}\text { Content } \\
\text { Oblivio } \\
\text { us }\end{array}$ & $\begin{array}{c}\text { Partially } \\
\text { Content } \\
\text { Aware }\end{array}$ \\
\hline $\begin{array}{c}\text { Delivery } \\
\text { Probability }\end{array}$ & Low & Medium & $\begin{array}{c}\text { Very } \\
\text { Low }\end{array}$ & High \\
\hline Average Latency & Low & Medium & High & $\begin{array}{c}\text { Very } \\
\text { Low }\end{array}$ \\
\hline Overhead Ratio & High & Medium & $\begin{array}{c}\text { Very } \\
\text { Low }\end{array}$ & Low \\
\hline RAM for JVM & Less & Less & Less & More \\
\hline
\end{tabular}


From Figure 3 it is found that the delivery probability of MaxProp is higher than that of PRoPHET, Epidemic and Spray-and-Wait respectively. The average latency of Spray-and-Wait is higher than that of PRoPHET, Epidemic and MaxProp. The overhead ratio of Spray-and-Wait is smaller than that of MaxProp, PRoPHET and Epidemic respectively. MaxProp performs well as compared to all other three protocols based upon the comparison factors but it may slow down the simulations due to large number of nodes and frequent encounters. Finally, MaxProp is having high delivery probability and low average latency. So we can calculate the traffic offloading performance of MaxProp. At first we predict that all nodes compete in the message broadcasting process, but only segments of all the nodes are concerned in every data. Update interval defines how many neighbors of nodes identified in last update.

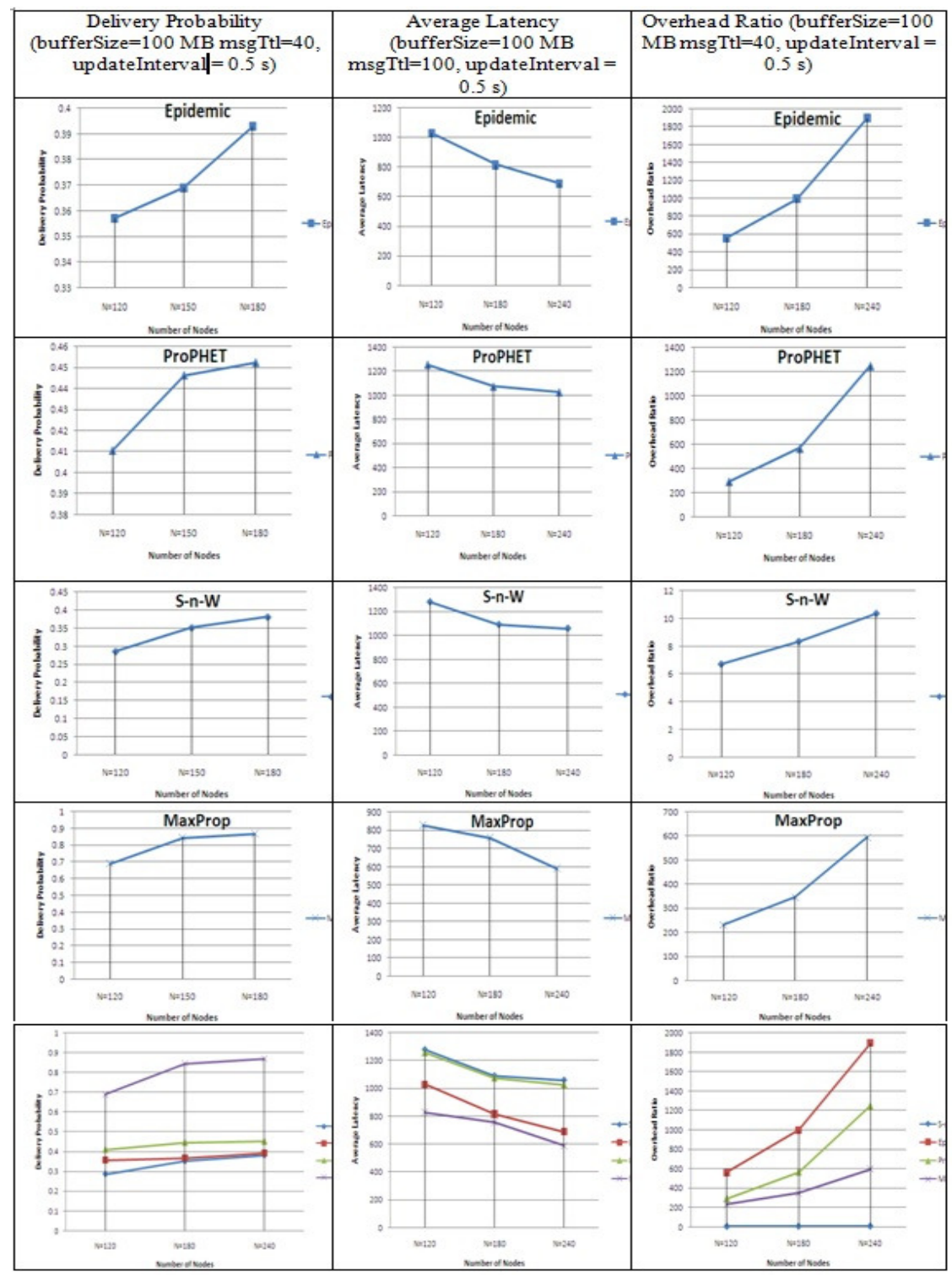

Figure 3: Comparison of Delivery Probability, Average Latency and Overhead Ratio of Epidemic, PRoPHET, Spray-andWait and MaxProp under different number of nodes 
Figure 4 shows the traffic offloading ratio under various update intervals. Traffic offloading ratio can be calculated as the ratio between the numbers of nodes delivered the message successfully to the total number of nodes. It can be inferred from the figure that traffic offloading ratio maximizes with the increasing of update interval from 0.02 to 2 seconds. The traffic offloading ratio of MaxProp is $90 \%$ when the update interval is 2 seconds. If the update interval is 0.02 seconds, the traffic offloading ratio is $63.33 \%$. MaxProp routing protocol can offload $90 \%$ of cellular or mobile network traffic.

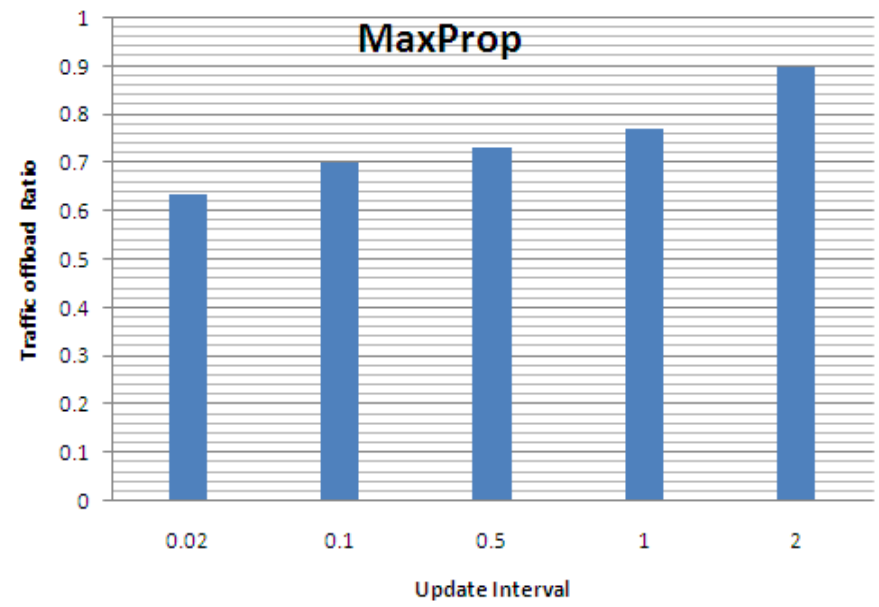

Figure 4: Traffic offloading ratio under different update interval

\section{CONCLUSION}

In this paper we mainly focused on opportunistic routing protocols in order to offload mobile data efficiently and effectively in cellular network through Subscribe-and-Send architecture. With offloading solution, information service provider can decrease cellular messages and thus reducing their operational cost. The main focus of our study is purely performance oriented. We discussed MaxProp, Spray-and-Wait, Epidemic and evaluate their traffic offloading performance, delivery probability, average latency and overhead ratio through ONE simulator. The simulation demonstrated that MaxProp routing protocol performs best followed by all other protocols. The simulation results show that Subscribe-and-Send architecture with MaxProp can offload mobile data traffic effectively. By applying this offloading technology we can reduce the bandwidth load and save the bandwidth cost. Our preliminary experimental results show that Subscribe-and-Send architecture with MaxProp can offload $90 \%$ of the cellular network or mobile data traffic.

\section{REFERENCES}

[1] Wikipedia, "Mobile data offloading". [Online]. Available: https://en.wikipedia.org /wiki/ Mobile_data_offloading.

[2] ComputerWeekly, "Smartphones and tablets drive mobile data traffic up 23\%, says Gartner". [Online]. Available: http://www.computerweekly.com/news/2240105255/Smartphones-and-tabletsdrive-mobile-data-traffic-up-23-says-Gartner.

[3] Cisco, "Cisco Visual Networking Index: Global Mobile Data Traffic Forecast Update 2014-2019 White Paper". [Online]. Available: 
International Journal of Wireless \& Mobile Networks (IJWMN) Vol. 10, No. 1, February 2018

http://www.cisco.com/c/en/us/solutions/collateral/serviceprovider/visual-networking-indexvni/white_paper_c11-520862.html.

[4] Cisco Visual Networking Index: Global Mobile Data Traffic Forecast Update 2014-2019 White Paper

[5] Choudhary, Kaushik, "An Overview of Opportunistic Communication".

[6] Bo Han; Pan Hui; Kumar, V.S.A.; Marathe, M.V.; Jianhua Shao; Srinivasan, A., "Mobile Data Offloading through Opportunistic Communications and Social Participation", Mobile Computing, IEEE Transactions on , vol.11, no.5, pp.821-834, May 2012.

[7] Lu Xiaofeng; Hui Pan; Lio, P., "Offloading mobile data from cellular networks through peer-to-peer WiFi communication: A subscribe-and-send architecture", Communications, China, vol.10, no.6, pp.35-46, June 2013.

[8] A. Balasubramanian, R. Mahajan, and A. Venkataramani, "Augmenting Mobile 3G Using WiFi", Proc. ACM MobiSys, pp. 209-222, June 2010.

[9] "Line2 iPhone application", [online]. Available: http://www.line2.com

[10] “iPassConnect”, [online]. Available: http://www.ipass.com

[11] "MOTO: Mobile Opportunistic Traffic Offloading", D1.1 - Project Presentation by MOTO, February 2013.

[12] Vahdat, Amin, and David Becker, "Epidemic routing for partially connected ad hoc networks", Technical Report CS-200006, Duke University, 2000.

[13] Lindgren, Anders, Avri Doria, and Olov Schelén, "Probabilistic routing in intermittently connected networks", ACM SIGMOBILE mobile computing and communications review 7.3, pp.19-20, 2003.

[14] Spyropoulos, Thrasyvoulos, Konstantinos Psounis, and Cauligi S. Raghavendra, "Spray and wait: an efficient routing scheme for intermittently connected mobile networks", Proceedings of the 2005 ACM SIGCOMM workshop on Delay-tolerant networking, ACM, 2005.

[15] Burgess, John, et al., "MaxProp: Routing for Vehicle-Based Disruption-Tolerant Networks", INFOCOM Vol. 6. 2006.

[16] Keränen, Ari, Jörg Ott, and Teemu Kärkkäinen, "The ONE simulator for DTN protocol evaluation", ICST, 2009. 